\title{
Phenomenological uncertainties imposed by our local magnetized neighborhood on the interpretation of the UHECR directional data
}

\author{
Thomas Fitoussi* \\ Instituto de Ciencias Nucleares, UNAM, Circuito Exteriror S/N, Ciudad Universitaria, México \\ D. F. 0.4810, México \\ E-mail: thomas.fitoussi@correo.nucleares.unam.mx
}

\section{Gustavo Medina-Tanco}

Instituto de Ciencias Nucleares, UNAM, Circuito Exteriror S/N, Ciudad Universitaria, México

D. F. 0.4810, México

E-mail: gmtanco@correo.nucleares.unam.mx

\section{Juan-Carlos D'Olivo}

Instituto de Ciencias Nucleares, UNAM, Circuito Exteriror S/N, Ciudad Universitaria, México D. F. 0.4810, México

\begin{abstract}
Magnetic fields can produce non-negligible deflections in the trajectories of Ultra-High Energy Cosmic Rays (UHECR), hindering the identification of potential sources from their observed arrival directions. The Galactic magnetic field is rather well understood inside the Galactic disk both in structure and topology, and its effects can be deconvolved with some degree of confidence. The extragalactic magnetic field poses a much more complicated challenge, but considerable effort has been devoted to assessing its effects on UHECR propagation for a variety of models and assumptions. The halo magnetic field, on the other hand, has been largely neglected in the literature. Our current knowledge of the halo magnetic field is extremely limited and we do not know with certainty its intensity, extension or, even more relevant for UHECR propagation, its topology and extension. In the present work, we use numerical simulations to analyze different halo magnetic field models and their impact on the interpretation of UHECR directional data. We show that the effects can be dramatic in some cases, highlighting the importance of taking them into account when evaluating the true uncertainty intrinsic to many phenomenological analyses.
\end{abstract}

36th International Cosmic Ray Conference -ICRC2019-

July 24th - August 1st, 2019

Madison, WI, U.S.A.

\footnotetext{
* Speaker.
} 


\section{Introduction}

Since they are charged particles, UHECR are deflected by the ambient magnetic fields in the different astrophysical regions: extragalactic and Galactic magnetic field (GMF). The extragalactic magnetic field is poorly known. Depending on its origin (primordial or plasma ejections from galaxies), the theoretical constraints can be really different (for a review, see [3]). Although attempts to measure this field (Faraday rotation of distant blazars, cosmological electromagnetic cascades) have not been successful so far, upper and lower limits have been imposed on it. Its amplitude is expected to be between $10^{-9}$ and $10^{-17} \mathrm{G}$ for a coherence length ranging from a few kpc to the size of the Universe. On the other hand, the GMF is quite well-known inside the Galactic disk [5, 6]. Nevertheless, despite considerable efforts to measure and model its different components, a full understanding of the global structure of the GMF is still missing (see, e.g., [7, 8] and references therein). This is particularly true with regard to the long-range behavior: both the amplitude and structure of the magnetic field in the halo are almost unknown.

A complete parameterization of both the regular and random components of the magnetic field in our Galaxy is essential in order to predict the pattern of UHECR deflections, identify sources and gain insight into composition. A way to study the propagation of UHECR in the local magnetized neighborhood is to use magnetic lenses as described in Bretz et al. [2]. The basic principle is to backtrack anti-protons from Earth to the border of the Galaxy for a given model of the Galactic magnetic field. Using a pixelization of the sky following the scheme of HEALpix [4], we can convert with these lenses a probability map of arrival directions outside the Galaxy into a probability map of observed arrival directions.

In this work, we built different magnetic lenses for different models of the regular component of the GMF. We consider the models for the disk and the halo considered by Pshirkov et al. [10], complemented with an extra component we introducefor the halo magnetic field. To illustrate the importance of the halo magnetic field on the directional observation of UHECR we apply this magnetic lensing to the case of the full-sky blazars emission at different energies.

\section{Galactic magnetic field modeling}

We apply the model presented in Ref. [10] as it is implemented in CRPropa 3 [1]. The components of the magnetic field in cylindrical coordinates are

$$
B_{\theta}=B(r, \theta, z) \cos p, \quad B_{r}=B(r, \theta, z) \sin p, \quad B_{z}=0 .
$$

The two versions of the model are specified by the following expressions of $B(r, \theta, z)$ :

- Assymetrical (ASS) model

$$
B_{A S S}(r, \theta, z)=B(r)\left|\cos \left(\theta-b \ln \frac{r}{R_{\odot}}+\phi\right)\right| e^{-|z| / z_{0}},
$$


- Bisymetrical (BSS) model

$$
B_{B S S}(r, \theta, z)=B(r) \cos \left(\theta-b \ln \frac{r}{R_{\odot}}+\phi\right) e^{-|z| / z_{0}},
$$

with $b=\tan ^{-1} p, \phi=b \ln \left(1+d / R_{\odot}\right)-\pi / 2$, and

$$
B(r)= \begin{cases}B_{0} \frac{R_{\odot}}{r \cos \phi}, & r>R_{c}, \\ B_{0} \frac{R_{\odot}}{R_{c} \cos \phi}, & r<R_{c}\end{cases}
$$

The definitions and values of the parameters in the previous formulas are[10]:

$$
\begin{array}{llr}
R_{\odot} & \text { distance from Sun to the center of the Galaxy } & 8.5 \mathrm{kpc} \\
z_{0} & \text { typical altitude of attenuation } & 1 \mathrm{kpc} \\
B_{0} & \text { initial magnetic field amplitude } & 2 \mu \mathrm{G} \\
R_{c} & \text { Radius of the central region where disk field is constant } & 5 \mathrm{kpc} \\
d & \text { distance to the first reversal } & -0.6 \mathrm{kpc} \\
p & \text { pitch angle } & -5^{\circ} \\
& & -6^{\circ}
\end{array}
$$

Above the magnetic field in the arms of the Galaxy, the model incorporates a halo component, which consists of a purely toroidal field whose direction in the southern hemisphere is reversed with respect to the one in the northern hemisphere:

$$
B_{\theta}^{H}(r, z)=\operatorname{sign}(z) B_{0}^{H}\left[1+\left(\frac{|z|-z_{0}^{H}}{z_{1}^{H}}\right)^{2}\right]^{-1} \frac{r}{R_{\odot}} e^{1-r / R_{\odot}},
$$

with the following definition and value of the different parameters [10]:

$$
\begin{array}{llrl}
B_{0}^{H} & \text { initial halo magnetic field amplitude } & 4 \mu \mathrm{G} & \\
z_{0}^{H} & \text { vertical position of the halo } & 1.3 \mathrm{kpc} & \\
z_{1}^{H} & \text { vertical scale of the halo } & 4 \mathrm{kpc} & \left(|z|<z_{0}^{H}\right) \\
& & 0.25 \mathrm{kpc} & \left(|z|>z_{0}^{H}\right) \\
R_{0}^{H} & \text { horizontal scale of the halo } & 8 \mathrm{kpc} &
\end{array}
$$

In this model, the magnetic field does not extend more than a few kpc above or below (north and south) the Galactic plane. On the contray, the halo can extend farther than $20 \mathrm{kpc}$ (pehaps up to $100 \mathrm{kpc})$.

On the other hand, as it is well known, part of the Sun's magnetic field is carried by the solar wind plasma, filling the heliosphere completely. The global structure of the heliospheric magnetic field is well described by the Parker spiral model [9]. A similar effect associated with the rotation of the Galaxy as a whole could produce an additional component of the magnetic field that extends long distances in the Galactic halo. To circumvent the mentioned limitation, we complement the previous model by adding a Parker like component to the halo magnetic field. In spherical coordinates, this field is given by

$$
B_{\rho}^{P}(r, \theta, z)=\left(\frac{\rho_{0}}{\rho}\right)^{2} B_{r}\left(\rho_{0}\right), \quad B_{\theta}^{P}(r, \theta, z)=\left(\frac{\rho_{0}}{\rho}\right)^{3} B_{\theta}\left(\rho_{0}\right), \quad B_{\phi}^{P}(r, \theta, z)=\left(\frac{\rho_{0}}{\rho}\right) B_{\phi}\left(\rho_{0}\right)
$$


where $\rho$ is the radial component of the position and $\rho_{0}$ is the corresponding radial position of the limit of the Galactic plan such as $\rho_{0}=\rho z_{0} / z . B_{r}\left(\rho_{0}\right), B_{\theta}\left(\rho_{0}\right)$ and $B_{\phi}\left(\rho_{0}\right)$ are the component of the Pshirkov et al. [10] at the border of the Galaxy in $\rho_{0}$.

\section{Impact on sources observations}

To highlight the impact of the halo magnetic field, we simulated four different sources located at $25 \mathrm{Mpc}$ in different direction of the sky. The sources are considered emitting isotropically iron nuclei at $200 \mathrm{EeV}$. The results of interactions (photo-pion production, photo-disintegration, nuclear decay) and transport (no extragalactic magnetic field) is detected when they reach a sphere of 100 kpc around the center of the Galaxy.

To model the deflection due to the Galactic and halo magnetic field, we used the lensing technique presented in Bretz et al. [2]. We built our own magnetic lens by backtracking anti-protons following the magnetic field implemented at the previous section. The limit on the extension of the halo is put by limiting the size of the sphere around the center of the Galaxy up-to which we backtrack particles $(20,40,100 \mathrm{kpc})$. The color scale gives the energy of the cosmic rays.

On figure 1 is presented the result of the deflection of cosmic rays by the two different Galactic magnetic field models (ASS left column and BSS right column) and different halo magnetic field extension (from top to bottom none, $20 \mathrm{kpc}, 40 \mathrm{kpc}, 100 \mathrm{kpc}$ ). Each map shows the arrival direction of cosmic rays from four different sources with different positions in the sky. The blue area around the sources shows the arrival direction of all cosmic rays from this source outside the Galaxy. Cosmic rays are identified by their source of origin: source 1 purple dots, source 2 green crosses, source 3 orange triangle and source 4 red pentagons. The color scale depicts the energy of cosmic rays (log scale).

Two aspect of the magnetic field are impacting the arrival direction of the cosmic rays: the modeling of the arm magnetic field (ASS or BSS) and the extension of the halo magnetic field.

The first line of figure 1 shows the impact of the different model of Galactic arm magnetic field: ASS (left) and BSS (right). Source 3 (in the left bottom quarter of the map) is almost not affected by the changing of modeling. Source 4 (right bottom quarter), in the case of the BSS model, is separated in three spots with one appearing in the northern hemisphere. The source 1 also shows a second spot for the BSS model. The source 2 located at the Galactic center is the most affected by the model of magnetic field inside the arms.

Adding the extra halo component up to $20 \mathrm{kpc}$ drastically change the arrival direction of cosmic rays for sources located at the Galactic and anti-Galactic center (source 1 and 2), both for ASS and for BSS. In particular, for source 1 a secondary component appears in the southern hemisphere for the ASS model. Surprisingly, this secondary component only exists for a halo of $20 \mathrm{kpc}$. If the halo extends further $(40,100 \mathrm{kpc})$, then this component disappears. The source 2 is especially affected in the BSS case where the events are closer to the source. Increasing the halo size, bring the event closer. The source 4 is really affected if the halo extend beyond $30 \mathrm{kpc}$ and start to exhibite specfic 

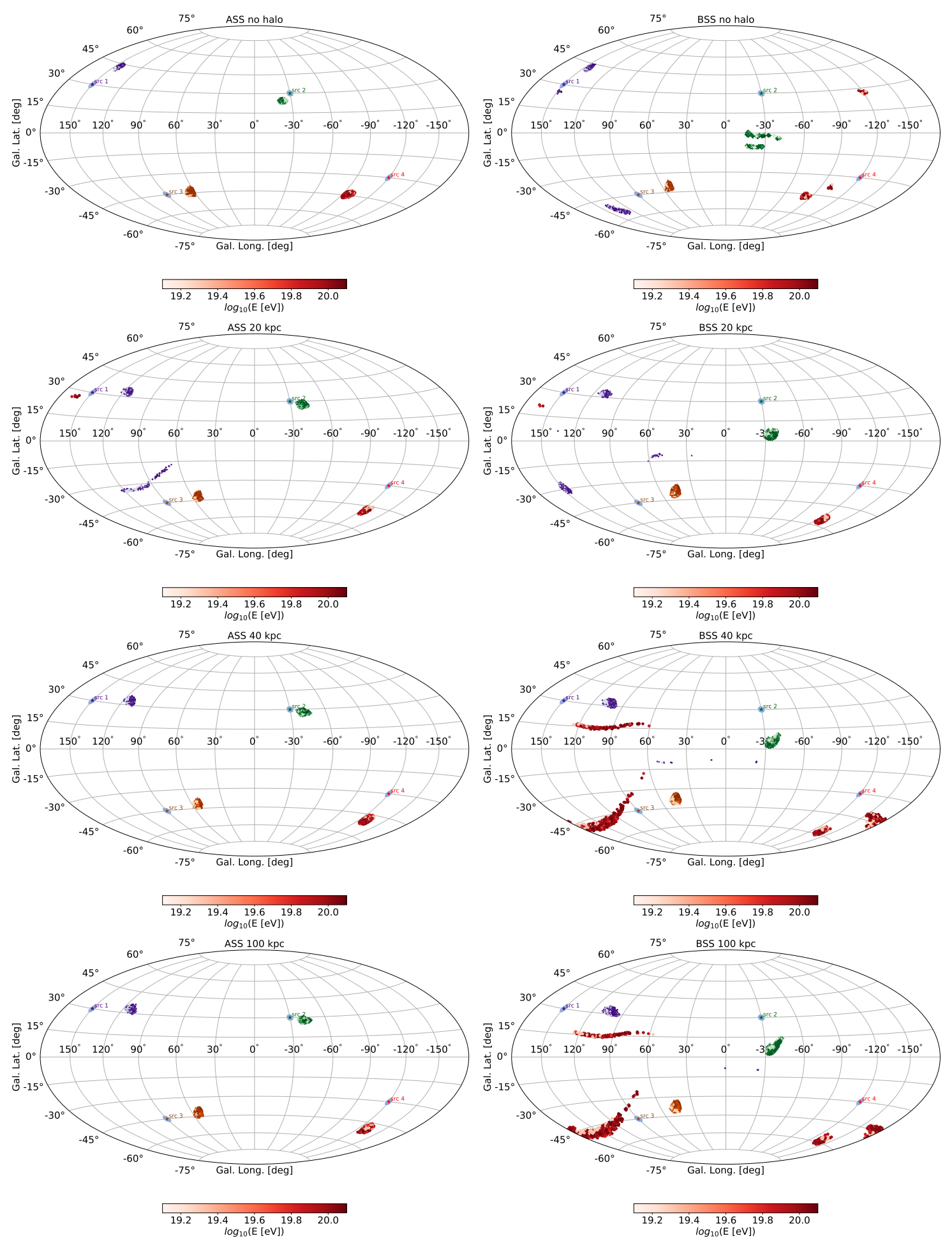

Figure 1: Arrival direction of cosmic rays for four different sources. Blue area around each source represents the arrival directions of all cosmic rays on the exterior of the Galaxy. Purple points, green crosses, orange triangles and red pentagons represent cosmic rays after being deflected by the Galactic and halo magnetic field for, respectively source 1, 2, 3 and 4. Color scale represents the energy in log scale. Left: ASS model, right: BSS model. From top to bottom: no halo, halo extending up-to 20, 40, $100 \mathrm{kpc}$. 
patterns in the sky. Finally, source 3 is barely affected by the configuration of the magnetic field in the Galaxy and the halo.

\section{Acknowledgements}

The authors acknowledge the support of this work through grant PAPIIT IN 103207, and CONACyT CB 239660 and the technical assistance of Mtro. Juan Luciano Díaz González with computational cluster infrastructure. T. F. thanks the financial support of DGAPA-UNAM.

\section{References}

[1] R. A. Batista, A. Dundovic, M. Erdmann, et al. CRPropa 3 - a public astrophysical simulation framework for propagating extraterrestrial ultra-high energy particles. J. Cosmol. Astropart. Phys., 2016(5):038, 2016. ISSN 1475-7516. doi: 10.1088/1475-7516/2016/05/038. URL http://stacks.iop.org/1475-7516/2016/i=05/a=038.

[2] H.-P. Bretz, M. Erdmann, P. Schiffer, D. Walz, and T. Winchen. PARSEC: A parametrized simulation engine for ultra-high energy cosmic ray protons. Astroparticle Physics, 54:110 117, 2014. ISSN 0927-6505. doi: 10.1016/j.astropartphys.2013.12.002. URL http:// adsabs.harvard.edu/abs/2014APh...54 ..110B.

[3] R. Durrer and A. Neronov. Cosmological magnetic fields: their generation, evolution and observation. The Astronomy and Astrophysics Review, 21(1):62, 2013. ISSN 0935-4956, 1432-0754. doi: 10.1007/s00159-013-0062-7.

[4] K. M. Gorski, E. Hivon, A. J. Banday, B. D. Wandelt, F. K. Hansen, M. Reinecke, and M. Bartelmann. HEALPix: A framework for high-resolution discretization and fast analysis of data distributed on the sphere. ApJ, 622(2):759-771, 2005. ISSN 0004-637X. doi: 10.1086/427976. URL https://doi.org/10.1086\%2F427976.

[5] J. L. Han, R. N. Manchester, A. G. Lyne, G. J. Qiao, and W. van Straten. Pulsar rotation measures and the large-scale structure of galactic magnetic field. The Astrophysical Journal, 642(2):868-881, 2006. ISSN 0004-637X, 1538-4357. doi: 10.1086/501444. URL http: //arxiv.org/abs/astro-ph/0601357.

[6] M. Haverkorn. Magnetic fields in the milky way. arXiv:1406.0283 [astro-ph], 407:483-506, 2015. doi: 10.1007/978-3-662-44625-6_17.

[7] R. Jansson and G. R. Farrar. The galactic magnetic field. ApJL, 761(1):L11, 2012. ISSN 2041-8205. doi: 10.1088/2041-8205/761/1/L11. URL http://stacks.iop.org/ $2041-8205 / 761 / i=1 / a=L 11$.

[8] R. Jansson and G. R. Farrar. A new model of the galactic magnetic field. ApJ, 757(1):14, 2012. ISSN 0004-637X. doi: 10.1088/0004-637X/757/1/14. URL http://stacks . iop.org/0004-637X/757/i=1/a=14. 
[9] E. N. Parker. Dynamics of the interplanetary gas and magnetic fields. 128:664, 1958. ISSN 0004-637X, 1538-4357. doi: 10.1086/146579. URL http: / / adsabs . harvard.edu/ doi/10.1086/146579.

[10] M. S. Pshirkov, P. G. Tinyakov, P. P. Kronberg, and K. J. Newton-McGee. Deriving the global structure of the galactic magnetic field from faraday rotation measures of extragalactic sources. ApJ, 738(2):192, 2011. ISSN 0004-637X. doi: 10.1088/0004-637X/738/2/192. URL http://stacks.iop.org/0004-637x/738/i=2/a=192. 